\title{
SLOSH-FREE POSITIONING OF CONTAINERS WITH LIQUIDS AND FLEXIBLE CONVEYOR BELT
}

\author{
Peter Hubinský ${ }^{*}$ - Thomas Pospiech ${ }^{* *}$
}

\begin{abstract}
This paper describes a method for slosh-free moving of containers with a liquid at which the conveyor belt is flexible. It shows, by means of experimental results, that a container filled with a liquid can be regarded as a damped pendulum. Upon parameter identification of the two single-mode subsystems, theoretical modelling of the complete system is described. With respect to industrial application, feedforward control is used to transfer the container in horizontal direction without sloshing. The applied method requires deterministic and hard real time communication between the PLC and the servo amplifier which is realized here with Ethernet POWERLINK. The principle structure, calculations, time duration and the robustness of the basic types of input shaper are described and compared with each other. Additionally the positioning results of the two-mode system are presented. Finally, a possibility of the principle software implementation is presented.

K e y w ords: liquid container transfer, elimination of residual vibration, two-mode system, input signal shaping
\end{abstract}

\section{INTRODUCTION}

If state-of-the-art filling systems for liquid food as for example milk or yogurt are regarded, it stands out that those systems are operated in a fully automated way and the complete working process is running without any impact of persons. The process is controlled by numerous sensors and various actuators.

For the actual function of these systems, two main requirements must be fulfilled next to the procedural challenges as for example cleaning the container before the liquid is filled in: On the one hand, the container must be filled with the corresponding liquid and on the other hand it must be sealed afterwards. In industrial application, two basic concepts for an operation mode are distinguished from each other: continuous and discontinuous operation.

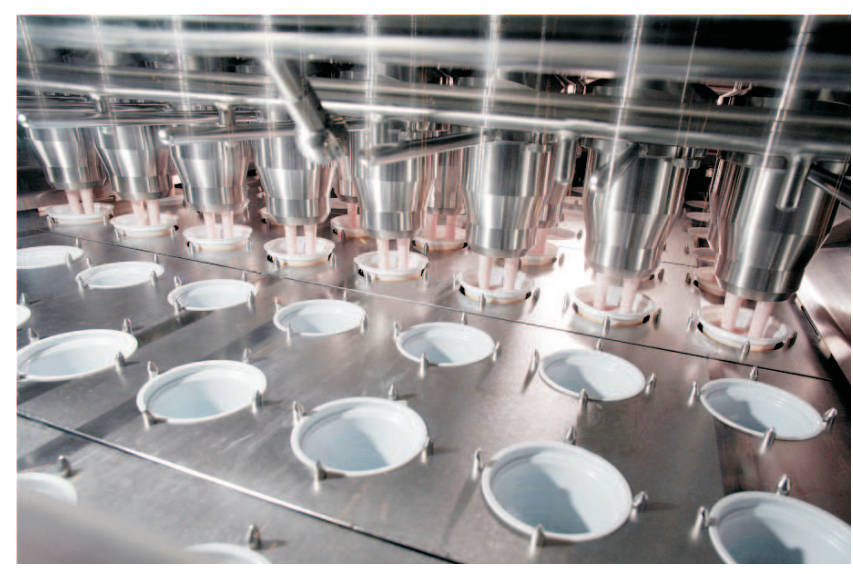

Fig. 1. Discontinuous filling plant [OYSTAR Gasti]

During continuous operation, the containers are conveyed on conveyor belts. The single working stations or production steps, as for example container cleaning, filling and sealing do not stop the conveyor belt iethey are carried along with the containers. The mechanical and electrical efforts of such machines are significant and thus correspondingly cost-intensive.

With discontinuous (clocked) machines, the above mentioned operation steps are static. The conveying belt with the containers must be stopped and started for every filling sequence.

This modular and economic design is opposed by a critical operational range. After the containers have been filled with the corresponding liquid, several operation sequences pass by until they are sealed. The critical area is the result of starting and stopping the conveyor belt which leads to the liquids in the containers being initiated to move. If this movement becomes too heavy, the liquid spills over the containers as a reaction of itself and the oscillating conveying belt. As soon as the cup seam is contaminated (in this case by the liquid), the cap cannot be sealed any more onto the cups respectively the containers cannot be hermetically sealed. Apart from that, Fig. 1 indicates that this operational area is sterile and therefore spilled liquid would imply increased cleaning efforts. In addition, the implementation of sensors in this area of the machine is impossible - this means that the only possibility will be a feed-forward control method. On the other hand a maximum production output is always aspired, which leads to a minimum process (moving) period. Together with the actual filling procedure (feeding the liquid into the container), this time is the longest process period with those kinds of lines.

The specified problem can therefore be summarized as follows: Directly after stopping the conveyor belt, the liquid and the conveyor belt must be calm which means

\footnotetext{
* Slovak University of Technology in Bratislava, Slovakia, Faculty of Electrical Engineering and Information Technology, Institute of Control and Industrial Informatics, peter.hubinsky@stuba.sk, ${ }^{* *}$ Faculty of Electronics and Mechanics, Heilbronn University, Max-PlanckStr. 39, 74081 Heilbronn, pospiech@hs-heilbronn.de
} 


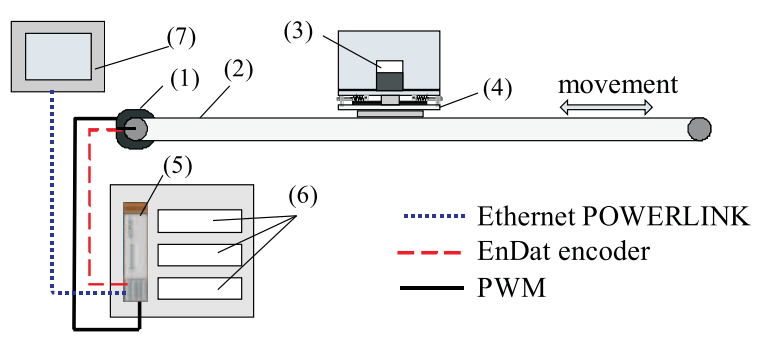

Fig. 2. Scheme of experimental set-up: (1) - Servo-motor with encoder and gear, (2) - Linear axis, (3) - Container with liquid, (4) Runner with oscillating skid, (5) - Servo amplifier (ACOPOS 1022), (6) - Electro-technology, (7) - Power Panel (PLC with visualization)

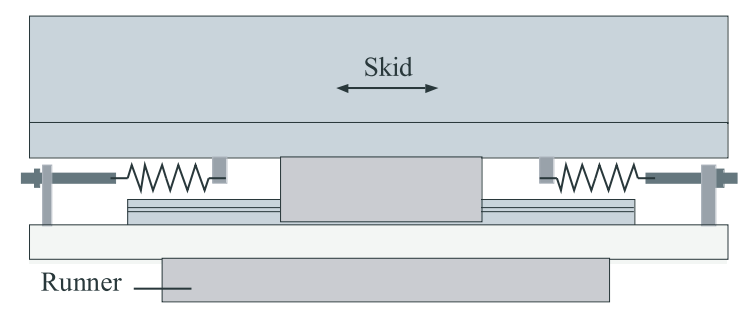

Fig. 3. Reproduction of the oscillation conveyor belt

they must not perform any movements (resonance!). Furthermore, a maximum deflection of the liquid within the container at a minimum process period must not be exceeded. These tasks should be solved without measuring the liquid surface or the vibration of the conveying belt during the process.

\section{A BENCH-SCALE MODEL}

The experimental set-up represents the "practical foundation for model verification, parameter identification and testing of control methods.

This test set-up has a total length $l$ of $2500 \mathrm{~mm}$ and allows for traverse paths of at most $1900 \mathrm{~mm}$. In order to achieve the highest level of precision and dynamics with positioning or traversing, all utilised components are purchased from leading industrial manufacturers. The communication between the PLC and the servo amplifier is $2 \mathrm{~ms}$. This means, in this cycle time the servo amplifier gets new reference values from the PLC via Ethernet POWERLINK. The positioning of the runner is indirectly measured by the encoder system of the servo motor. Deformations of the toothed belt in the linear axis utilised here can be neglected. That means, also the actual velocity and acceleration are calculated in the servo amplifier based on the signal from the encoder system. The accuracy of the complete electromechanical part is about $\pm 0.1 \mathrm{~mm}$.

The oscillation of the conveyor belt (eg chain) is reproduced here by an additional skid on a linear bearing, centred with two springs (Fig. 3).
By replacing the springs with correspondingly stiff elements the positioning task is automatically reduced to a single mode oscillating system (meaning non-flexible conveyor belt).

As for the measurement of the liquid surface and the position of the skid, a laser-triangulation sensor is used. The sensors send the analogue data (4-20 mA) to a PLC in a cycle time of $2.5 \mathrm{~ms}$, meaning $400 \mathrm{~Hz}$ sampling rate. The resolution of measuring the deflections is $0.1 \mathrm{~mm}$ (for the liquid and the skid).

\section{MODEL ANALYSIS}

This chapter describes how the complete system is modelled and how its parameters are identified. For this purpose, it is assumed that the conveyor belt with the filled containers can be approximated by the combination of two single mode systems: a linear singular-mass oscillator for the transport unit and a damped pendulum-model for the dynamic behaviour of the liquids in the containers. Related works [1] and [2] show that liquid sloshing in a container can be approximated as a two-dimensional phenomenon if the observed liquids possess the following characteristics:

- no modification of viscosity caused by temperature $T$, pressure $p$, time $t$,

- density $\rho$ is constant (incompressible liquid),

- Newton-fluid (no shearing stress occurs).

A further assumption is that no deformations occur at the outside walls of the container, ie the geometry of the containers is stiff and not flexible.

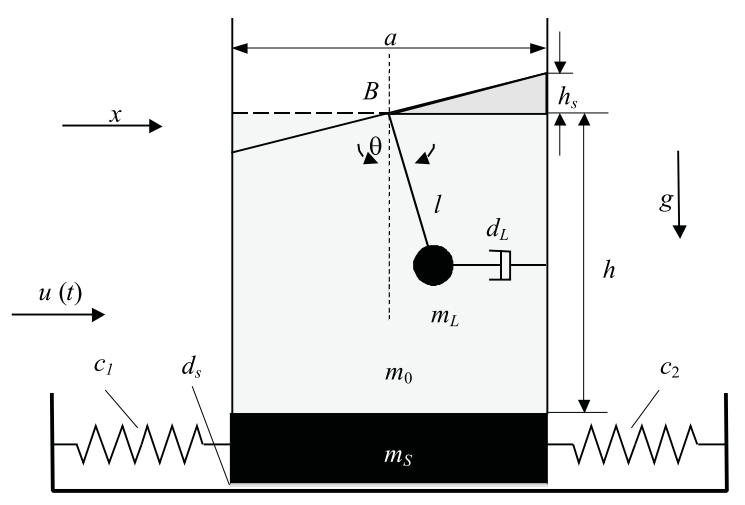

Fig. 4. Combination of the single systems

Figure 4 shows the sketch of the assumed model and is pointing out the geometrical correlations and the characteristic parameters for the equations of motion (for abbreviations see Table 1).

According to [1] and [2], the total mass $m$ of the liquid can be divided into an oscillatory mass part $m_{L}$ and a "fixed" mass part $m_{0}$. Thus, it applies for a rectangular container

$$
m=m_{0}+m_{L}, \quad m_{L}=m \frac{1}{\pi^{3}} 8 \frac{a}{h} \tanh \left(\pi \frac{h}{a}\right) .
$$




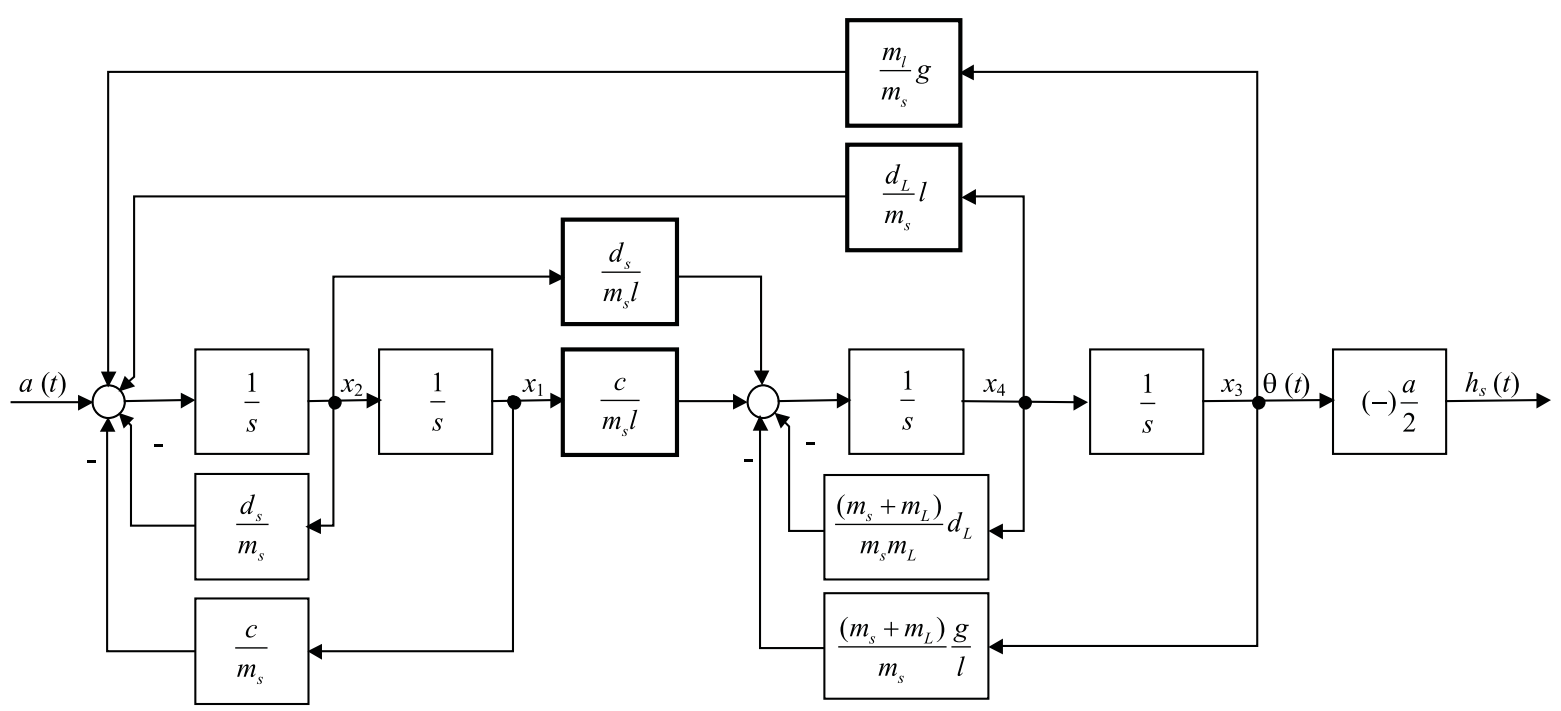

Fig. 5. Block-schema of the complete system ( drawn directly from the state-space description)

It can be shown that the system can now be described via two subsequent (here already linearized) differential equations of second order by establishing the angular momentum balance and the horizontal pulse balance

$$
\begin{gathered}
\ddot{x}_{s}=-\frac{d_{S}}{m_{S}} \dot{x}_{S}-\frac{c}{m_{S}} x_{S}+\frac{d_{L}}{m_{S}} l \dot{\theta}+\frac{m_{L}}{m_{S}} g \theta+a(t), \\
\ddot{\theta}=-\frac{m_{S}+m_{L}}{m_{S} m_{L}} d_{L} \dot{\theta}-\frac{m_{S}+m_{L}}{m_{S}} \frac{g}{l} \theta+\frac{d_{S}}{m_{S} l} \dot{x}_{S} \\
+\frac{c}{m_{S} l} x_{S} .
\end{gathered}
$$

By establishing the state variables, those two differential equations can be converted into four differential equations of first degree and can thus be expressed in the more compact form of a state space description. The state variables result in

$$
\begin{aligned}
& x_{1}=x_{S}, \quad \dot{x}_{1}=x_{2}, \\
& x_{2}=\dot{x}_{S}, \quad \dot{x}_{2}=\ddot{x}_{S}, \\
& x_{3}=\theta, \quad \text { and } \quad \dot{x}_{3}=x_{4} \text {, } \\
& x_{4}=\dot{\theta}, \quad \dot{x}_{4}=\ddot{\theta} .
\end{aligned}
$$

Equations (2) and (3) then pass into

$$
\begin{aligned}
\dot{x}_{1} & =x_{2}, \\
\dot{x}_{2} & =-\frac{c}{m_{S}} x_{1}-\frac{d_{S}}{m_{S}} x_{2}+\frac{m_{L}}{m_{S}} g x_{3}+\frac{d_{L}}{m_{S}} l x_{4}+a, \\
\dot{x}_{3} & =x_{4}, \\
\dot{x}_{4} & =\frac{c}{m_{S} l} x_{1}+\frac{d_{S}}{m_{S}} x_{2}-\frac{m_{S}+m_{L}}{m_{S}} \frac{g}{l} x_{3} \\
& -\frac{m_{S}+m_{L}}{m_{S} m_{L}} d_{L} x_{4} .
\end{aligned}
$$

Thus, the state space form in the time domain, in consideration of the linearized correlation between the deflection $h_{s}$ and the angle $\theta$ (compare figure 4) for the output vector $\boldsymbol{c}^{\top}$ is

$$
\begin{aligned}
& \dot{\boldsymbol{x}}(t)=\mathbf{A} \boldsymbol{x}(t)+\mathbf{B} \mathbf{u}(t), \\
& \boldsymbol{y}(t)=\boldsymbol{c}^{\top} \boldsymbol{x}(t) .
\end{aligned}
$$

In this formula, the system matrix $\mathbf{A}$ and the input vector $\boldsymbol{b}$ as well as the output vector $\boldsymbol{c}$ are time-invariant and merely depend on the model parameters. The dynamic characteristics are (only) included in system matrix A and can be calculated via the eigenvalues of $\mathbf{A}$ by finding the solution for the characteristic equation. Based on the linear transformation

$$
(\lambda \mathbf{I}-\mathbf{A}) \mathbf{x}=0
$$

A nontrivial solution $(\boldsymbol{x} \neq 0)$ only exists if

$$
\operatorname{det}(\lambda \mathbf{I}-\mathbf{A})=0 \text {. }
$$

For the present $4 \times 4$ system matrix, this characteristic equation provides four conjugate complex zero points which correspond to the poles of the transfer functions in the range. On the other side the poles of a transfer function are specifying the system characteristics eigen frequency and damping, how the following equations are pointing out

$$
\begin{aligned}
& \lambda_{1,2}=-D_{1} \omega_{n, 1} \pm j \omega_{n, 1} \sqrt{1-D_{1}^{2}} \\
& \lambda_{3,4}=-D_{2} \omega_{n, 2} \pm j \omega_{n, 2} \sqrt{1-D_{2}^{2}}
\end{aligned}
$$

A further and helpful overview can be given by a blockschema of the system (Fig. 5), which can be directly drawn from (9) and considering the corresponding correlation between the deflection $h_{s}$ and the angle $\theta$.

Figure 5 shows clearly the coupling of the two single systems by the appropriately highlighted blocks and without any problems the transfer functions of the singlesystems can be derived through this.

The (homogenous) differential equations for the two separated single mode systems can be described as follows.

$$
\begin{aligned}
\ddot{x}+\frac{d_{S}}{m_{S}} \dot{x}+\frac{c}{m_{S}} x & =0, \\
\ddot{\theta}+\frac{d_{L}}{m_{L}} \dot{\theta}+\frac{g}{l} \theta & =0 .
\end{aligned}
$$




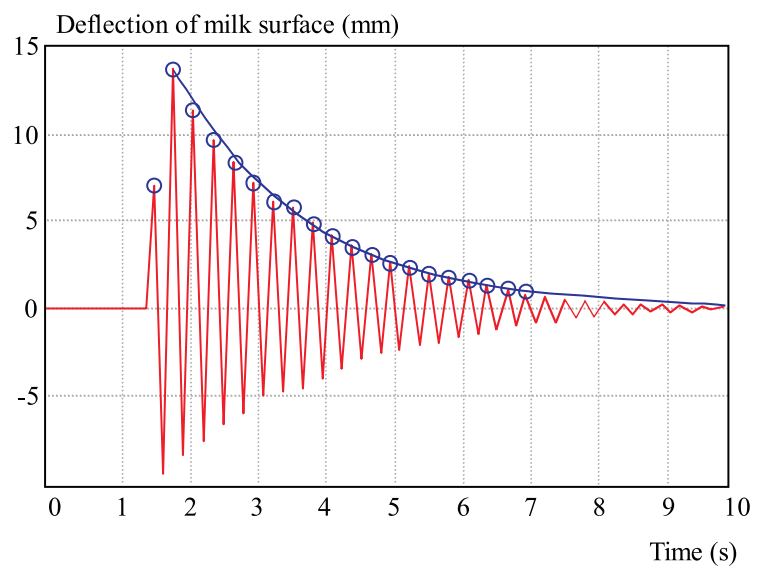

Fig. 6. Measured impulse response of the liquids surface (milk)

Table 1. Parameters of the skid- and liquid-subsystem

\begin{tabular}{|c|c|c|c|}
\hline Symbol & Quantity & Value & Unit \\
\hline$m_{s}$ & Mass of the skid ${ }^{1}$ & 4.8 & $\mathrm{~kg}$ \\
\hline$c$ & Spring constant ${ }^{2}$ & 4274 & $\mathrm{~N} / \mathrm{m}$ \\
\hline$d_{s}$ & Damping coefficient & 7.16 & $\mathrm{~kg} / \mathrm{s}$ \\
\hline$D_{s}$ & Damping skid & 0.025 & (1) \\
\hline$\omega_{n, S}$ & Natural Angular frequency ${ }^{3}$ & 29.84 & $\mathrm{rad} / \mathrm{s}$ \\
\hline$\omega_{d, S}$ & Damped Angular frequency ${ }^{3}$ & 29.83 & $\mathrm{rad} / \mathrm{s}$ \\
\hline$m$ & Liquid's mass & 0.448 & $\mathrm{~kg}$ \\
\hline$m_{L}$ & Liquid's oscillating mass & 0.087 & $\mathrm{~kg}$ \\
\hline$m_{0}$ & Liquid's fixed mass & 0.361 & $\mathrm{~kg}$ \\
\hline$m_{c}$ & Container's mi & 0.08 & $\mathrm{~kg}$ \\
\hline$a$ & width of conts & 0.06 & $\mathrm{~mm}$ \\
\hline$h_{S}$ & Filling level & 0.08 & $\mathrm{~kg} / \mathrm{s}$ \\
\hline$d_{L}$ & Damping coefficient & 0.089 & $\mathrm{~kg} / \mathrm{s}$ \\
\hline$D_{L}$ & Damping liquid & 0.024 & - \\
\hline$\omega_{n, L}$ & Natural Angular frequency ${ }^{4}$ & 21.43 & $\mathrm{rad} / \mathrm{s}$ \\
\hline$\omega_{d, L}$ & Damped Angular frequency ${ }^{4}$ & 21.42 & $\mathrm{rad} / \mathrm{s}$ \\
\hline$g$ & Acceleration of gravity & 9.81 & $\mathrm{~m} / \mathrm{s}^{2}$ \\
\hline$l$ & Pendulum length & 0.021 & $\mathrm{~m}$ \\
\hline$\omega_{1}$ & $1^{\text {st }}$ Eigen frequency & 21.12 & $\mathrm{rad} / \mathrm{s}$ \\
\hline$D_{1}$ & Damping & 0.024 & - \\
\hline$\omega_{2}$ & $2^{\text {nd }}$ Eigen & 28.84 & $\mathrm{rad} / \mathrm{s}$ \\
\hline$D_{2}$ & Damping & 0.025 & - \\
\hline
\end{tabular}

${ }^{1}$ measuring equipment included

${ }^{2}$ representing spring constant $\left(c=c_{1}+c_{2}\right)$

${ }^{3}$ Skid-system

${ }^{4}$ Liquid-system

For solving (9) or (10) and (11) all system parameters must be noted. Some of them (eg masses of skid, liquid and container and the width of the container in movement direction) can be measured directly without any complexity. Others like damping $\left(d_{s}\right.$ and $\left.d_{L}\right)$, spring constant $c$ and the (virtual) length of the pendulum $l$ can be determined by some experimentally tests in form of analysing the step or impulse responses of the separated single systems.

By establishing the decay coefficient $\delta$ and the eigen angular frequency $\omega_{n},(10)$ and (11) can be brought to the following (global) form

$$
\ddot{y}+2 \delta \dot{y}+\omega_{n}^{2} y=0 .
$$

The general solution for this differential equation is

$$
y(t)=C e^{-\delta t} \sin \left(\omega_{d} t+\varphi_{d}\right)
$$

in which it is

$$
\begin{aligned}
C & >0, \\
0 & \leq \varphi_{d} \leq 2 \pi, \\
\omega_{d} & =\sqrt{\omega_{n}^{2}-\delta^{2}} .
\end{aligned}
$$

The periodic time $T_{d}$ of the system is

$$
T_{d}=\frac{2 \pi}{\omega_{d}} .
$$

Equation (13) gives the oscillation amplitude at point of time $t$, whereas for one periodic time later it can also be stated

$$
\begin{aligned}
& y\left(t+T_{d}\right)=C e^{-\delta\left(t+T_{d}\right)} \sin \left(\omega_{d}\left(t+T_{d}\right)+\varphi_{d}\right), \\
& y\left(t+T_{d}\right)=C e^{-\delta\left(t+T_{d}\right)} \sin \left(\omega_{d} t+\varphi_{d}\right) .
\end{aligned}
$$

Taking the logarithm of the relation of two subsequent amplitudes with the same deflection directions, results in the logarithmic decrement $\Lambda$.

$$
\ln \frac{y(t)}{y\left(t+T_{d}\right)}=\delta T_{d}=\Lambda .
$$

By establishing Lehrs attenuation factor $D$ it follows for (16)

$$
\begin{aligned}
D & =\frac{\delta}{\omega_{n}}, \\
\omega_{d} & =\omega_{n} \sqrt{1-D^{2}} .
\end{aligned}
$$

Thus, (20) can also be rewritten and so it follows for $D$ :

$$
D=\frac{1}{\sqrt{1+\left(\frac{2 \pi}{\Lambda}\right)^{2}}} .
$$

This means that at least two subsequent amplitudes (with the same deflection) must be known - in respect of time as well as in respect of their values. From this, the damping ratio $D$ and afterwards the corresponding eigen angular frequencies $\left(\omega_{n}\right.$ and $\left.\omega_{d}\right)$ and the decay coefficient $\delta$ can be calculated. Now it is no problem to calculate (with help of comparison the coefficients from (12), (10) and (11)) the missing system parameters in (5) for solving (8).

Figure 6 shows a velocity impulse response of a container filled with milk as liquid. The envelope of the oscillation has a form of $e^{-\delta t}$-function and is typical for a viscous friction.

Table 1 gives a summary of the system parameters and the characteristic values ( $\omega$ and $D)$ for the dynamic behaviour of the two single and the complete system (twomode).

The further course of this article now specifies the control method that eliminates the system natural oscillations and provides for improving the process. 


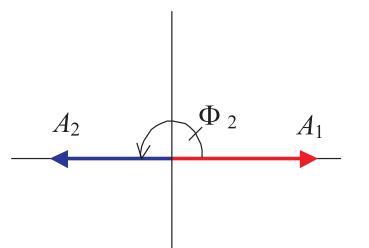

(a)

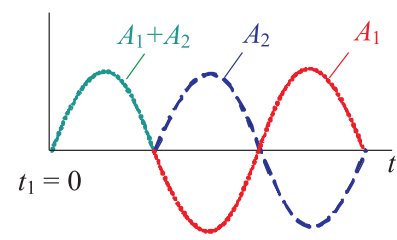

(b)

Fig. 7. Vector diagram and the impulse responses of a second order and damped oscillatory system
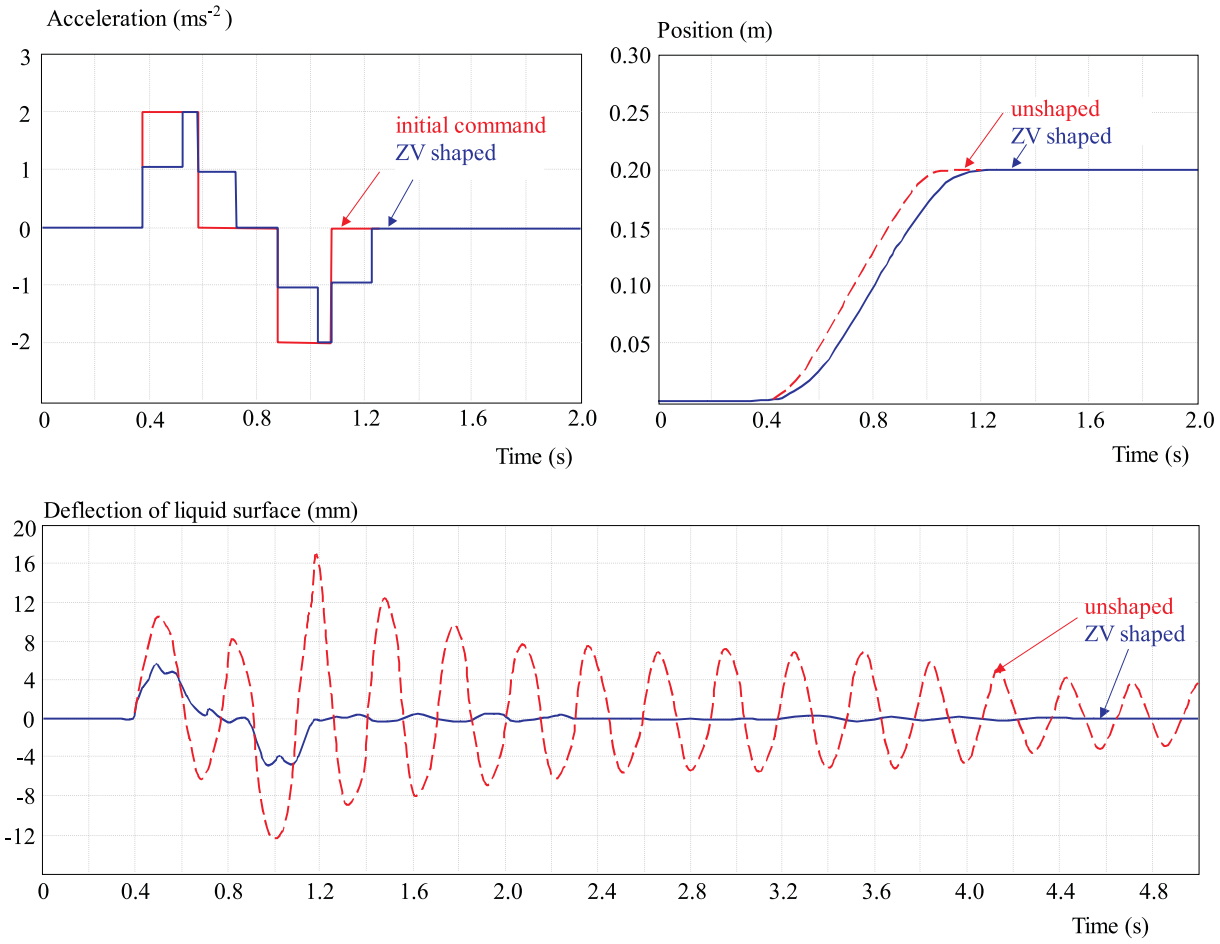

Fig. 9. Slosh-free positioning of the container filled with milk (single-mode) using ZV shaper $A_{1}=0.5698, A_{2}=0.4302 ; t_{1}=0 \mathrm{~s}$, $t_{2}=0.1467 \mathrm{~s}\left(\omega_{d L}=21.42 \mathrm{rad} / \mathrm{s}, D_{L}=0.024\right)$

\section{FEED-FORWARD CONTROL OF THE SYSTEM}

On the basis of the previous chapter, the eigen angular frequencies $\omega_{1,2}$ and the damping $D_{1,2}$ can be assumed as known. In the present chapter, the input shaper method is now applied to the system.

\subsection{Input Shaping for single-mode systems}

The basis of all algorithms in this area is spectral conditioning of the control signal in that way that any spectral component close to the natural frequency of the controlled mechanic system is eliminated. In turn, the idea of input shaping is to convolute the control signal at specific points of time by Dirac impulses. Figure 7 shows a vector diagram with two rotating impulses (pointers) and the corresponding impact of the impulses on an oscillatory system of second order.

After the second impulse, the overlapping of the two oscillations results in elimination so that no residual oscillations remain afterwards.

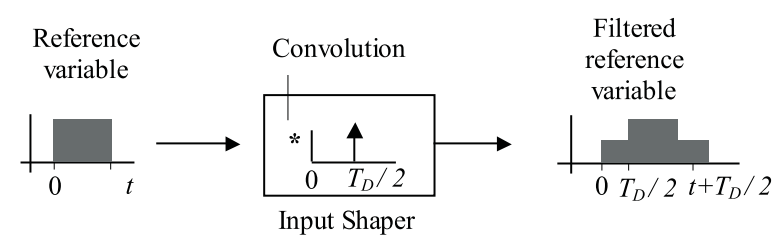

Fig. 8. Principle functionality of the ZV input shaper (deformation of the acceleration profile) 


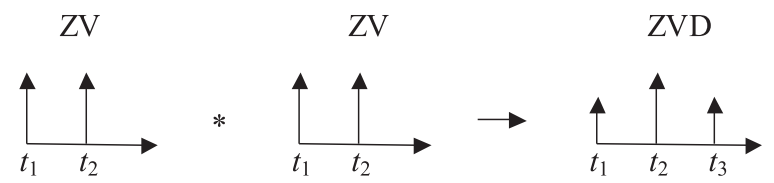

Fig. 10. Generation of the ZVD input shaper

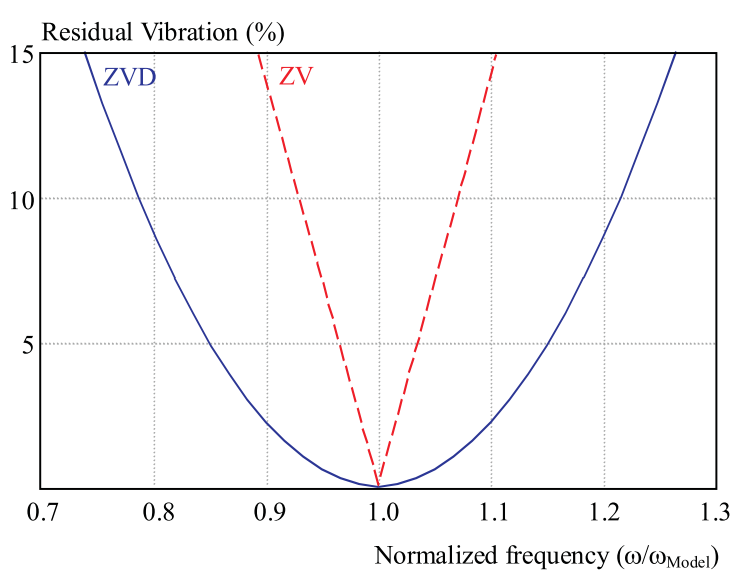

Fig. 11. Sketch of sensitive curve of ZV and ZVD shaper

of (23) and considering (24), the amplitude and time values for this input shaper result in

$$
A_{1}=\frac{1}{1+k}, A_{2}=\frac{k}{1+k}
$$
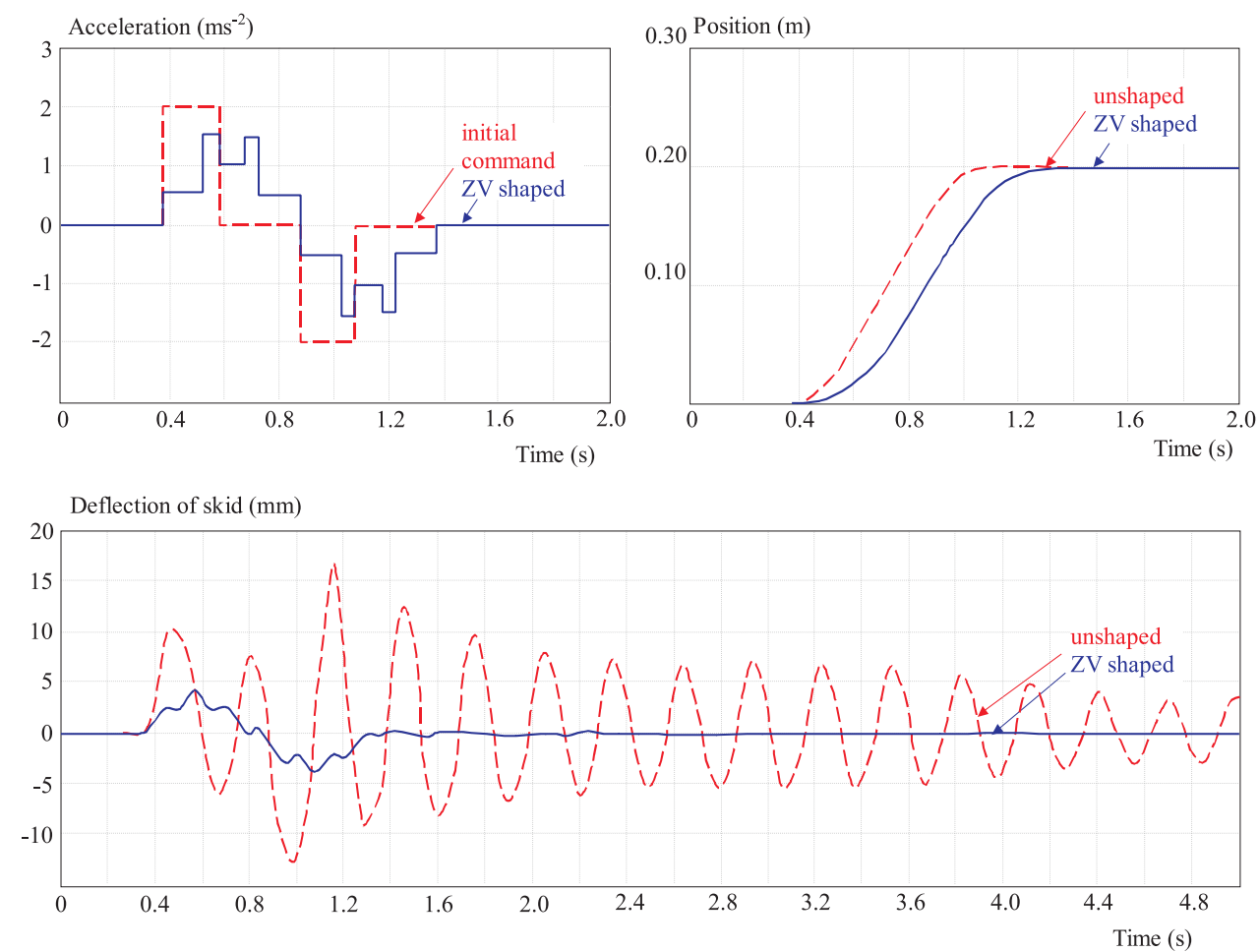

Fig. 12. Slosh-free positioning of the container filled with milk (single-mode) using ZVD shaper: $A_{1}=0.3247, A_{2}=0.4902, A_{3}=0.1851$;

$t_{1}=0 \mathrm{~s}, t_{2}=0.1467 \mathrm{~s}, t_{3}=0.2933 \mathrm{~s} ;\left(\omega_{d L}=21.2 \mathrm{rad} / \mathrm{s}, D_{L}=0.024\right)$

The amplitude and time values at last solely depend on the periodic time of the damped system $T_{D}$ and on the damping $D$. If now (any) input variable is convoluted by those two impulses, the oscillations will be eliminated after the second impulse due to the filtered reference variable. Figure 8 shows the principle functionality of the convolution.

If now the input signal, "filtered via the ZV shaper respectively acceleration profile is regarded, it turns out that the control period is prolonged against the original signal (here: rectangular shape) by half a periodic time.

Those diagrams show on the one hand the unshaped and ZV shaped acceleration profile and on the other hand the measured responses of the surface of the liquid. As the diagram shows, there is a definite improvement. The "residual oscillations with ZV control can be measured but are nearly invisible to the eye because they range within $<0.5 \mathrm{~mm}$. Those deflections of the liquid would never suffice in sloshing during clocked operation!

\subsubsection{Z V D Input Shaper}

According to [4], the ZVD input shaper is an extended version of the ZV shaper. In this respect, two interpretations form the basis for this theory. The ZVD input

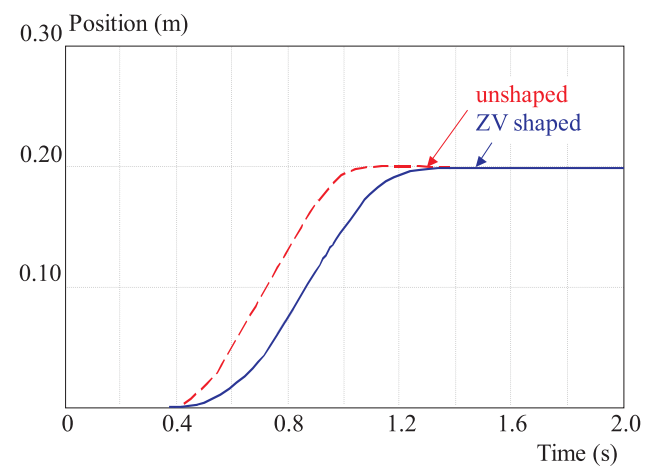




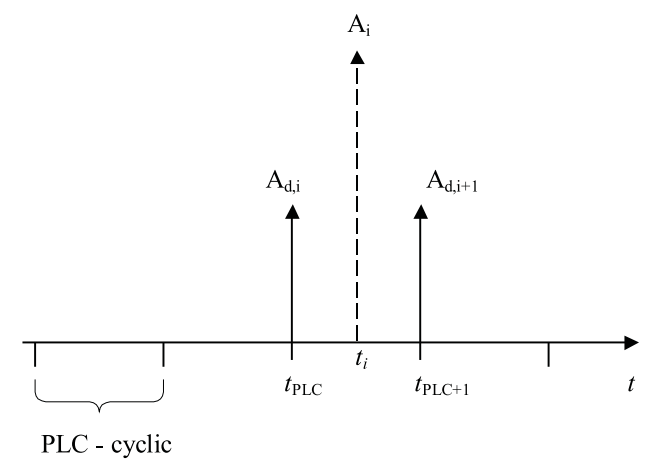

Fig. 13. Splitting an impulse at the PLC-cyclic points)
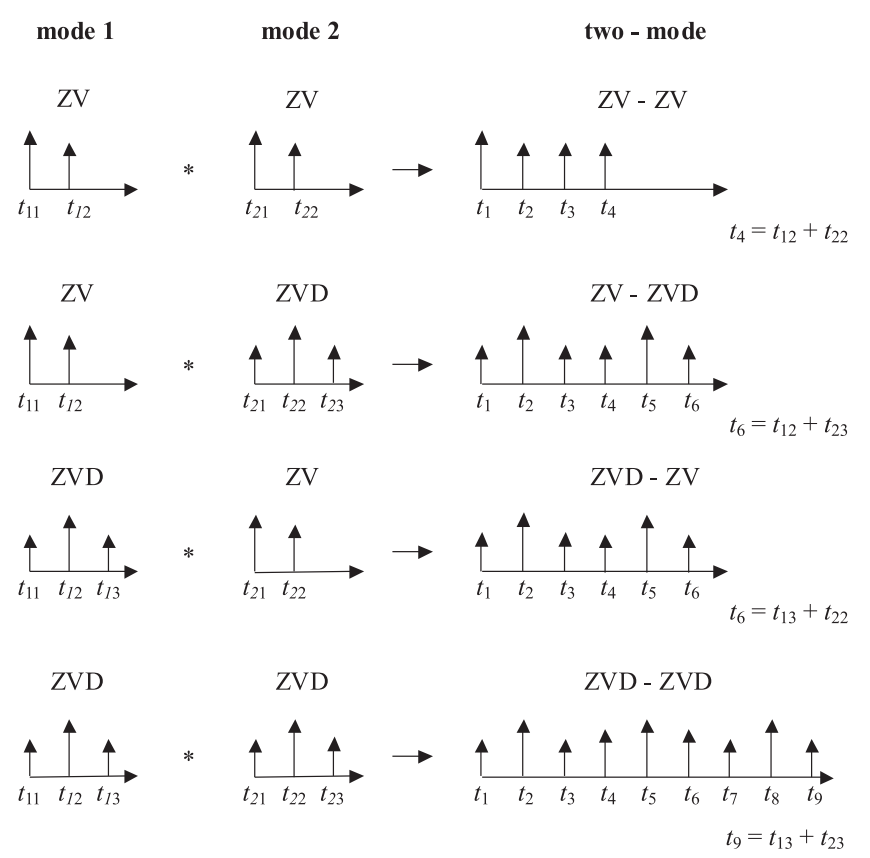

Fig. 14. Principle of generating multi-mode shaper with convoluted single mode shaper $\left(t_{6}^{*} \neq t_{6}^{*}\right)$ see Tab. 2 ; are not scaled!

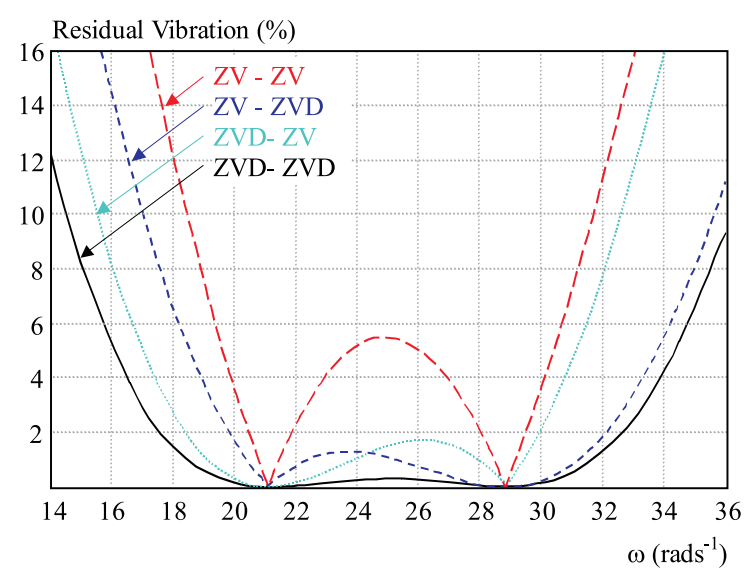

Fig. 15. Sensitivity curves of the convolved basic shapers

shaper receives its name from an additional condition (D for derivative $\rightarrow$ zero vibration and derivative).

$$
\frac{\mathrm{d}}{\mathrm{d} \omega} \sum_{i}^{n} A_{i} e^{-j D \omega_{n} t_{i}}=0 \text {. }
$$

In consideration of $(24),(27)$ results in three amplitudes with different amplitude values and time values

$$
A_{1}=\frac{1}{(1+k)^{2}}, A_{2}=\frac{2 k}{(1+k)^{2}}, A_{3}=\frac{k^{2}}{(1+k)^{2}}
$$

and

$$
T_{1}=, t_{2}=\frac{T_{D}}{2}, t_{3}=T_{D}
$$

A convolution by two identical ZV shapers gives a further interpretation of the present issue. Figure 10 is to point this out.

Equation (29) shows that, with the ZVD shaper, the control period is prolonged compared to the original reference input variable by the period $T_{D}$.

Figure 11 shows the sensitivity curves of the shapers described here. Those curves state the percentage residual oscillations of the system, depending on modelling errors in the eigen frequency.

This shows that the ZVD shaper has a more robust behaviour than the ZV shaper. The consequence is a shaper running time that is by $T_{D} / 2$ longer. The diagram below shows the ZVD shaper during positioning that corresponds to the shaper shown in Fig. 12 (initial command as comparison). Even if its behaviour leaves a more robust impression, the ZV shaper is likely to be preferred in the present case due to its shorter running time (which is automatically reflected in the total process time).

At first view, the acceleration profile after the ZVD shaper has a rather unusual shape. This is simply caused by the shapers running time that is longer than the positive or negative acceleration phase of the original reference variable. However, this does not have any impact on eliminating the oscillations.

\subsection{Discretization for implementation}

A continuous behaviour in respect of time was presumed for modelling as well as for calculating the single mode shaper. Depending on the systems periodic time $T_{d}$, the impulse times of the respective shaper can adopt any value. However, the principle function of a PLC is not continuous in respect of time but is time-discrete due to the adjusted cycle time. It is not very likely that the impulses are within the time scope of the PLC. In the present case, the servo amplifier (ACOPOS) "clocks the control unit via the here used bus system Ethernet POWERLINK. This means here that new reference values for acceleration can be given every $2 \mathrm{~ms}$. In this respect, it is possible to implement the input shaper because of the deterministic real-time behaviour of the bus-system (Buscycle). Reason is that the impulses must only be converted into the cycle time of the bus-system/PLC $t_{P L C}$, as Fig. 13 shows. 
A corresponding vector diagram shows that the following applies [9]

$$
A_{d, i}=\frac{A_{i} e^{-D\left(t_{P L C}-t_{i}\right) \omega_{n}} \sin \left(\Phi_{k+1}\right)}{\sin \left(\Phi_{k+1}\right) \cos \left(\Phi_{k}\right)+\sin \left(\Phi_{k}\right) \cos \left(\Phi_{k+1}\right)}
$$

and

$$
A_{d, i+1}=\frac{\mid A_{i} e^{-D\left(t_{P L C+1}-t_{1}\right.} \sin \Phi_{k}}{\sin \left(\Phi_{k+1}\right) \cos \left(\Phi_{k}\right)+\sin \left(\Phi_{k}\right) \cos \left(\Phi_{k+1}\right)}
$$

where,

$$
\begin{gathered}
\Phi_{k}=\left|t_{P L C}-t_{i}\right| \omega_{n} \sqrt{1-D^{2}}, \\
\Phi_{k+1}=\left|t_{P L C+1}-t_{i}\right| \omega_{n} \sqrt{1-D^{2}} .
\end{gathered}
$$

Thus, every impulse can be converted or adjusted to the utilized time pattern of the PLC cyclics via (30-33) and (24)! Thus, the single mode shaper that has been correspondingly digitalized can be implied on a control without any problems. The continuous ZVD shaper in Fig. 12 results after discretization with $t_{P L C}=0.002 \mathrm{~s}$ in

$$
\left[\begin{array}{c}
A_{i} \\
t_{i}
\end{array}\right]_{d Z V D}=\left[\begin{array}{ccccc}
0.3246 & 0.3273 & 0.1629 & 0.0619 & 0.1232 \\
0 & 0.146 & 0.148 & 0.292 & 0.294
\end{array}\right]
$$

It is important to note at this point that the above stated equations are in no case performed during the process running time (also not the equations of the corresponding shaper): The calculations are performed during development and thus do not pose any load on the control. The chapter about implementing clearly shows that the control method with the input shaper does not pose any challenge on the PLC and that the deterministic real time concept comes fully into effect! Of course it is the same with examining the following two-mode shapers. Even if single-mode shaping provides a solution for examining the single systems, the complete system would imply residual oscillations as long as the both damped eigen frequencies do not have exactly the same values (can normally not be assumed).

\subsection{Input shaping for multi-mode system}

Based on the above presented basic concept, a possibility is now shown of how the complete system can be positioned free from oscillations, Furthermore, the results received from the experimental set up are presented.

One possibility is to design two single mode shapers for the respective eigen frequencies, considering the related damping and to "fold" them with each other subsequently. This procedure can be compared to creating the ZVD shaper (please see Fig. 10). Depending on the required robustness, the shapers can be freely combined with each other. However the resulting number of impulses or the running time of the shaper should be considered in this process. Figure 14 emphasizes this principle correlation. However, please note that the respective two mode shapers are not discretized for clarity reasons.

The sensitivity curves of the above shown two-mode shaper are illustrated in Fig. 15 using the values out of Tab. $1\left(\omega_{1,2}, D_{1,2}\right)$.

The robustness at possible parameter variations during process time or with modelling errors in eigen frequency can be qualitatively compared with the sensitivity curves. The diagram shows that all four combinations eliminate both eigen frequencies. The ZVD-ZVD shaper clearly shows the most robust behaviour in covering a range of $15 \mathrm{rad} / \mathrm{s}<\omega<35 \mathrm{rad} / \mathrm{s}$ at which the residual oscillation is less than $8 \%$.Nevertheless, this combination has the longest running time by far, as Tab. 2 shows.

Table 2. Comparison: time of the last impulse

\begin{tabular}{ccc}
\hline Two-Mode shaper & Time of last impulse & Unit \\
\hline ZV-ZV $\left(t_{4}\right)$ & 258 & $\mathrm{~ms}$ \\
ZV-ZVD $\left(t_{6}\right)$ & 368 & $\mathrm{~ms}$ \\
ZVD-ZV $\left(t_{6}\right)$ & 408 & $\mathrm{~ms}$ \\
ZVD-ZVD $\left(t_{9}\right)$ & 516 & $\mathrm{~ms}$ \\
\hline
\end{tabular}

Note at this point that the input shapers do not have to be necessarily put on the corresponding eigen frequencies. The sensitivity curves show that different areas are covered by properly choosing the "given eigen frequency. This stands out when the ZV-ZVD shaper and the ZVD$\mathrm{ZV}$ shaper are compared to each other. The ZV-ZVD shaper is more robust between the two eigen frequencies. Furthermore, the running time in this case is approximately $40 \mathrm{~ms}$ shorter. The ZVD-ZV shaper on the contrary shows a better behaviour for frequencies below the first eigen frequency. This non-symmetric behaviour of the two kinds of shapers is caused by the existing periodicity.

The diagrams below display the positive results of the control concept presented here for the two-mode complete system.

The subsequent diagram shows a ZV-ZV shaped time optimal bang-bang acceleration profile $\left(a_{\max }=2.5 \mathrm{~m} / \mathrm{s}^{2}\right.$, $\left.t_{\text {bang }}=0.3 \mathrm{~s}\right)$ and the oscillation-free responses of the skid and the liquids surface.

\section{IMPLEMENTATION OF INPUT SHAPER}

The previous chapters presented a method of how to specify eigen frequencies by observing a model in theory and of how to use the findings for calculating the corresponding shapers. The present chapter is now to demonstrate how (almost) any shaper can possibly be realized. It is important to point out at this point once again that the previously stated calculations (modelling, etc) do not belong to the running time or to the tasks of the PLC program. All calculations are previously performed, 

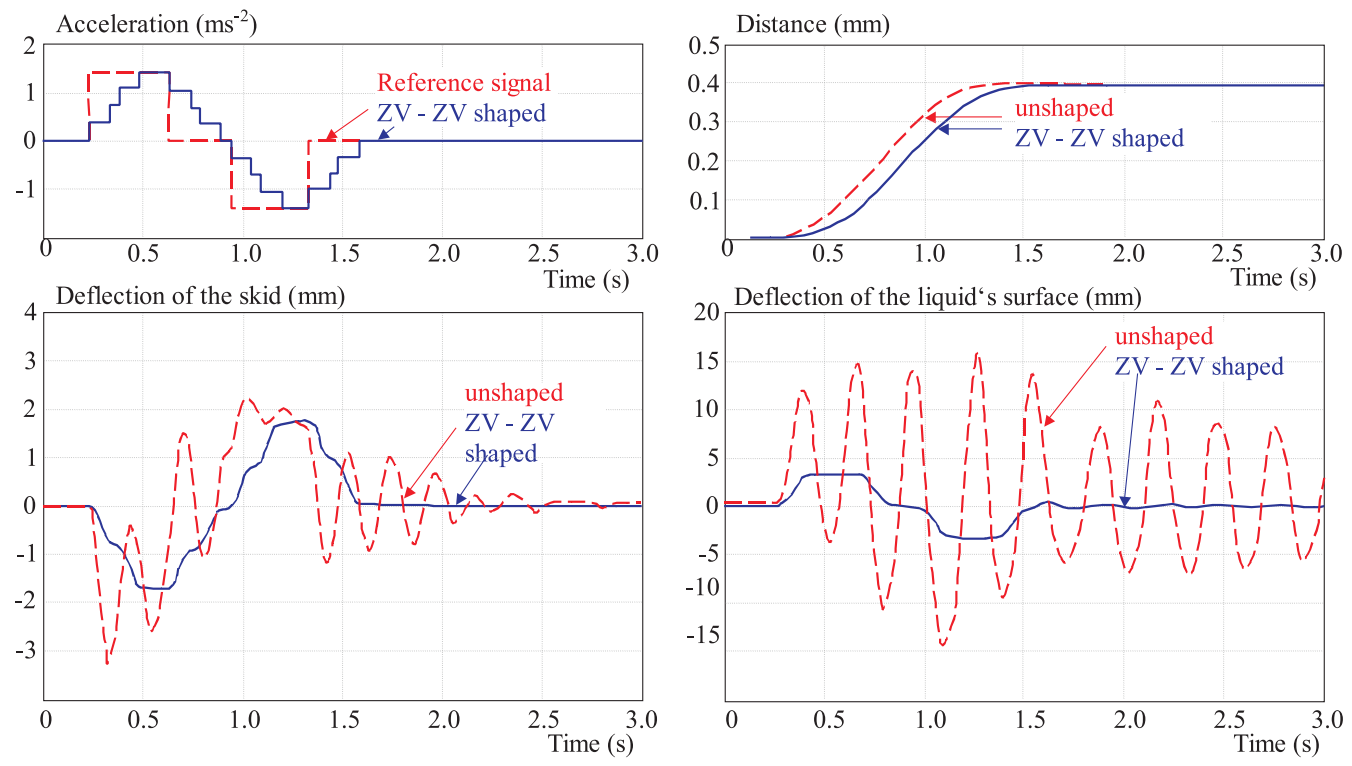

Fig. 16. Comparison of unshaped and ZV-ZV shaped responses; ZV-ZV: $A_{1}=0.3247, A_{2}=0.4902, A_{3}=0.1851, A_{4}=0.1851$; $t_{1}=0 \mathrm{~s}, t_{2}=0.109 \mathrm{~s}, t_{3}=0.149 \mathrm{~s}, t_{4}=0.258 \mathrm{~s}$
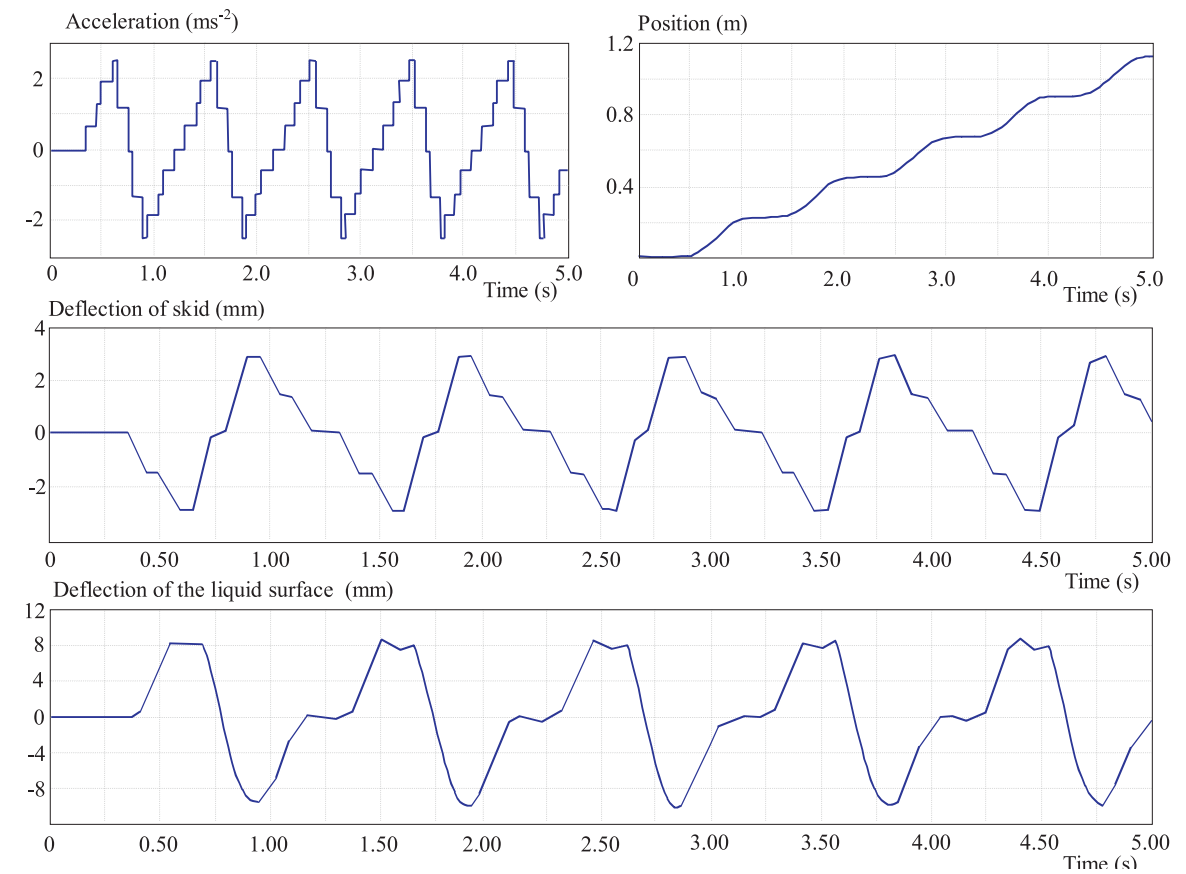

Fig. 17. Responses of the system; clocked positioning using bang-bang acceleration as reference (ZV-ZV shaped)

that means during developing. This also implies calculating the amplitudes and time values of the impulses of the corresponding shaper! The actual task of the PLC is "merely" convoluting the desired reference variable (acceleration) with the impulses of the shaper and to send the freshly generated reference variable "correctly" to the servo amplifier. In this respect, the term "convolution" mathematically means nothing else than the integral over two multiplied functions that are shifted in terms of time. In the present case, the acceleration $a(t)$ is one function and the other one is the Dirac pulse sequence of the input shaper.
The discrete convolution of $a(t)$ and the Dirac pulse sequence of the shaper is given by

$$
A(t) * \sum_{i} A_{i} \delta\left(t-t_{i}\right)=\sum_{i} A_{i} a\left(t-t_{i}\right)
$$

The discrete convolution does not pose any challenge on the PLC anyway. In fact, the principle operating mode of a control is virtually ideal for realization, because this procedure takes advantage of the deterministic and cyclic program operations. Figure 18 is to emphasize the basic idea of the approach performed here. 


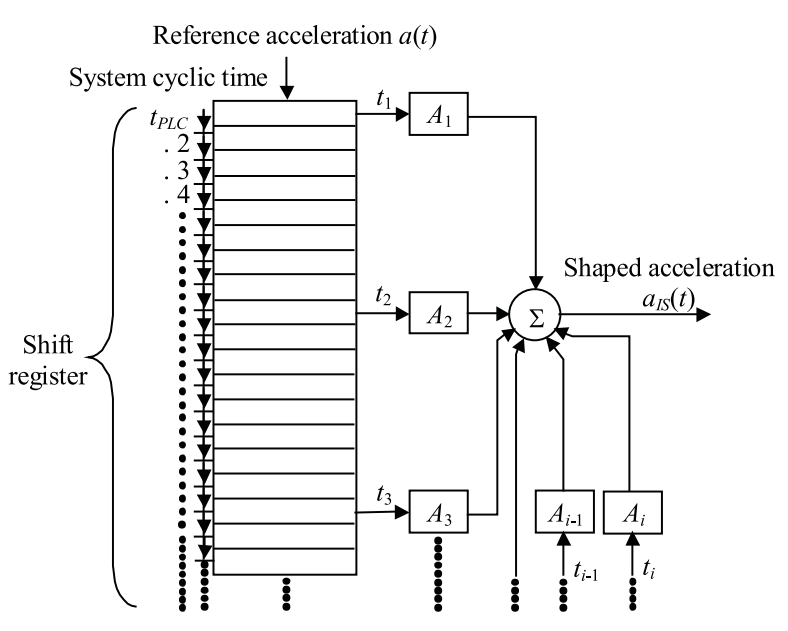

Fig. 18. Principle of implementation

Initially, the principle function is alike a shift register with fifo principle (first in first out). The shift register is clocked by the adjusted cycle time of the control. Afterwards, the respective elements of the shift register (depending on the points of time of the shapers impulses) must be multiplied with the related amplitudes coefficients and the multipliers must be added. This is absolutely no problem for almost every industrial control and it must not pose any challenge regarding the performance!

Furthermore it must be pointed out here that by the presented discretizing procedure, this method can also provide very good results for far longer cycle times than the $2 \mathrm{~ms}$, used in this case. Nevertheless one fact must not be overseen: even if the convolution is not a problem for the control, it must be considered that the convoluted reference variable must still be sent to the servo amplifier. The best calculations are worthless if the reference values cannot be received and processed within the "exact time pattern by the servo amplifier. The bus system is often the bottleneck in this regard or simply any clear deterministic behaviour is missing. The components utilized here, the PowerPanel 220 and the ACOPOS 1022 as a servo amplifier (both components made by Bernecker und Rainer) are linked with each other via the bus system Ethernet POWERLINK. Ethernet POWERLINK is a deterministically real time bus system that supplies optimum results for the demands made here.

\section{CONCLUSION}

The present article describes the possibilities of controlling open containers with liquids by using a flexible traction mechanism. Based on the industrial demands, a mathematical model is derived and the parameters are identified. Initially, the control method is merely described in terms of the single-mode subsystem (transport of a container filled with liquid). Later, the concept is extended to the two-mode complete system. By implementing on a test set up, the results gained from this are verified by means of measurement and are graphically displayed. Furthermore, the derived control methods are compared with each other in terms of robustness and running time. As a conclusion, the article states a possibility of how the control concept can be implemented on a PLC with a deterministically real time bus system.

\section{Acknowledgement}

The support provided by the grant VEGA 1/0690/09 is kindly acknowledged.

\section{REFERENCES}

[1] DODGE, F. T.: The New Dynamic Behaviour of Liquids in Moving Containers; remake of NASA SP106; Southwest Research Institute San Antonio, Texas; 2000.

[2] RAOUF, A. I. : Liquid Sloshing Dynamics, Cambridge University Press, 2005.

[3] LUTZ, H.-WENDT, W.: Taschenbuch der Regelungstechnik, 2. Auflage, Verlag Harri Deutsch, 1998. (in German)

[4] SINGHOSE, W. E.-SEERING, W.: Command Generation for Dynamic Systems, August 19, 2006, book on demand (www.lulu.com).

[5] HUBINSKÝ, P.-VRANKA, B.-JURIŠICA, L. : Genetic Algorithm Based Method of Elimination of Residual Oscillation in Mechatronic Systems, Kybernetika Journal (2005), 623-636.

[6] SINGHOSE, W.: Command Generation for Flexible Systems, MIT PhD-Thesis; S.B.M.E., Massachusetts Institute of technology, 1990 M.S.M.E., Stanford University, 1992.

[7] VITKO, A.-JURISICA, L.-KLUCIK, M.-DUCHON, F. : Context Based Intelligent Behaviour of Mechatronic Systems, Acta Mechanica Slovaca 12 No. 3-B (2008), 907916.

[8] HUBINSKÝ, P.—GRUHLER, G.-VRANKA, B. : Feedforward - Methods With Spectral Approach to Reduction of Oscillations in Mechatronic Systems, SPS/IPC/DRIVES Conference 2004 in Nürnberg.

[9] MURPHY, B. R.-WATANABE, I. : Digital Shaping Filters for Reducing Machine Vibration, IEEE Transactions on robotics and automotion 8 No. 2 (April 1992).

Received 6 September 2009

Peter Hubinský received the Engineer degree in technical cybernetics from the Faculty of Electrical Engineering of the Slovak University of Technology, Bratislava in 1985. In 1992 he received the $\mathrm{PhD}$ degree from the same University. He specialized further in system theory and drive technology and control of robotic systems and worked as an assistant at the Faculty of Electrical Engineering in this time. Since 1999 he has been Assoc. Professor at the Faculty of Electrical Engineering and Information Technology, Slovak University of Technology, Bratislava. His research and lecture activities are in the areas of servo systems, theory of dynamical systems, motion control systems, robotics and automation.

Thomas Pospiech received the Engineer degree in Mechatronics from Heilbronn University, Germany in 2002. From 2002 to 2005 he worked as an application engineer and project manager in software development for several industrial automation projects. Since 2005 he has been an external PhD student at the Faculty of Electrical Engineering and Information Technology, Slovak University of Technology, Bratislava. $\mathrm{He}$ is also a research associate for robotics and automation at Heilbronn University. His research interests are positioning techniques, process optimisation of servo controlled industrial applications, PLC programming and hardware design of microcontroller based PCBs. 